\title{
The 'Expiry Problem' of broad consent for biobank research - And why a meta consent model solves it
}

\section{The debate}

The best consent model for biobank research is the topic of ongoing discussion. In a previous article in JME we argued that a model of broad consent is faced with an 'expiry' problem. More specifically, we argued that a valid broad consent presupposes some form of mutual understanding between research participants and researchers of what it means to participate in biobank research falling within a certain category, e.g. "health-related research". Consenting to participate in such research is, because of the referential opacity of consent, only consent to the consent givers current understanding of "health-related research". . However, in the course of time research and social views may develop and move beyond the original, mutual understanding of what "health-related research" is. If this happens the initial broad consent cannot be considered valid any longer - it has expired. Neil Manson has recently sought to respond to this argument. He claims:

1) that changes in research methods do not make broad consent expire, because broad consent is consent to a complex governance structure protecting participants' interests

2) that the expiry problem also applies to meta consent because it includes broad consent as an option

So, on the one hand Manson sets out to salvage broad consent from the expiry argument. On the other hand, he claims that the meta consent model suffers from the same weakness. Despite the inconsistency 1 ) and 2) obviously cannot both be true - we shall in this brief reply show why they are both false. We argue:

1) that broad consent expires because of various types of changes, including changes in governance structures

2) that the dynamic and flexible nature of a meta consent model not only means that meta consent does not fall prey to the expiry argument - it solves it!

\section{The comprehensive expiry problem of broad consent}

Biobank research changes over time. Changes in how biobank research is conducted may significantly alter what research participation entails, and it may do so in ways that research participants could not anticipate at the time of providing consent. More specifically, there may be changes in (this is not an exhaustive list):
1) Methods
2) Purposes
3) Types of data used
4) Governance structures

The research methods are changing radically these years. As previously highlighted the advent of genetic sequencing methods have radically changed the nature of biobank research and research into hereditary diseases and genetic risk factors. The possibility of making a wide variety of incidental/secondary findings of direct and specific relevance for the future health of particular individuals and their families changes the relevance of biobank research for the individuals' lifestyle choices and life plans. It also generates highly personal and sensitive information about individuals and their families. These changes fundamentally alter the meaning and implications of research participation. Claiming by analogy, as Manson does, that changes 
in research methods make no more of a difference for an individual having provided tissue than someone having provided financial support, is blatantly false. Financial support is rarelyhighly personal and sensitive, and it very rarely reveals information about the donor's health or genetics. The financial information is simply not generative in the same way as a tissue sample is. And, there is furthermore also a parallel expiry problem in relation to targeted large donations. If someone in the early 1800s had given a large amount of money to phrenological research, the trustees disbursing the funding would have an expiry problem when it becomes clear that the phrenological research programme is defunct.

The research purposes also change. A recent study published in Science found that same-sex sexual behaviour is influenced by many genes. The study used genetic and other information from a number of sources, including UK Biobank. UK Biobank participants gave broad consent for "health-related research purposes". As we have argued elsewhere, conducting genetic studies of same-sex sexual behaviour on the basis of broad consent for health-related research purposes has two possible implications: 1) either it entails that the researchers and UK biobank considers same-sex sexual behaviour to be states of health or illness, or 2 ) it entails that genetic studies of any behaviour that may be correlated with a health-state or increase our understanding of human biology is health-related research. The former would be highly stigmatising (and is rejected by the authors). The latter would be a vast expansion of the scope of health-related research, since 1) nearly all behaviours have some correlation with health and 2) it would make all human genetic research "health-related". In either case the study implies a redefinition of "health-related research purposes" with wide-ranging implications for participation in biobank research.

The kind of data generated, linked to, and used in biobank research may also change as completely new data sources become available, or linkage becomes technically possible and easy. It is common for biobanks to get consent to linkage to and use of the participants' 'health records', but there is no obvious answer to whether or not the data from a participant's fitness tracker, or from participation in a social-media discussion group on psychosis is part of the 'health record'. Participants in older biobanks are very unlikely to have considered this when giving consent, and may now legitimately have different views on this. The biobank can therefore not claim to have consent to linkage to such data, even if both the researchers and the complex governance structure thinks that the data are now understood to be part of the health record.

The governance structure of biobanks may also change. Recently the Ethics and Governance Council of Biobank UK was superseded by the UK Biobank Ethics Advisory Committee. This was followed by a significant redefinition of the committee's role. From being tasked with acting "as an independent guardian of the UK Biobank Ethics and Governance Framework (EGF)" and monitoring and reporting "publicly on the conformity of the UK Biobank project with the EGF", the committee's role is now reduced to that of identifying and providing advice to the UK Biobank Board on relevant ethical issues. The independence and transparency of the ethical oversight have formally been toned down. Such changes in governance structures alters the conditions of research participation. Manson thinks that broad consent to biobank research is really consent to "a complex normative framework that protects the data subjects' interests, with independent monitoring ...". But even if broad consent is expanded to include consent to such "a complex network", it cannot reasonably be claimed to be consent to any possible future governance structure.

All of the changes above cannot reasonably be assumed to be included in the original mutual understanding of the scope of "health-related research". They are, we believe, changes that make the initial broad consent expire. Whether or he accepts this argument, Manson is caught in a dilemma here. Either he must maintain that there is no expiry problem - broad consent is consent to (almost) any change in methods, purposes, data types and governance structure. But then he is really defending blanket consent for biobank research and should openly admit to that. Or he must acknowledge the expiry problem, but is then left with the problem of adhering strictly to the scope of the initial broad consent.

\section{Why the expiry problem does not apply to a model of meta consent}


As originally conceived meta consent is a national or health care system-wide consent platform that allow citizens to decide how they would like to provide consent to future research use of different types of data, e.g. patient record data, registry data, genomic data; and revise those decision if and when they want to. In the meta consent model people are given the opportunity to decide for each of these types of data whether they in the future would like to provide i) consent to every specific research use of these data (specific consent), ii) consent to the research use of these data within broad categories of research (broad consent), or if they want to receive no future consent requests by up-front providing or refusing iii) consent to all future research (blanket consent/blanket refusal). The choices made would apply to biobank data, but also to, for instance secondary use of clinical data or social service data for research.

Manson thinks that if there is an expiry problem of broad consent, then it must also apply to a model of meta consent since it lets research participants opt for broad to future use of data. Manson fails, however, to appreciate a number of crucial differences between meta consent and broad consent:

1) Non-compulsory: At the point of providing meta consent, research participants can act on their concern for how research may change in the future. They can require consent requests for every specific use of their data. They are not forced to go with broad consent.

2) Flexible: Due to its digital implementation, research participants may at any point in time act on changes in research and revise their consent preferences. They are not forced to stick with an initial choice of broad consent for a particular type of data or a particular use.

3) Dynamic: Due to its digital implementation, a meta consent model incorporates a dynamic flow of information about research from researchers to the research participants (a feature from dynamic consent). Research participants will therefore know about on-going changes in research.

4) Precision: Due to its digital implementation that allows consent requests to be issued to research participants continuously, the research categories figuring in broad consent may be more specific, e.g. 'cardiovascular diseases', or 'cancer' instead of 'health-related research'

These features protect a meta consent model against the expiry problem. Research participants are empowered to act on unanticipated and unwanted changes in research they may previously have consented to. They are empowered to protect themselves against the expiry of their consent. And this is a moral reason to prefer meta consent over broad consent.

There is, however, another important difference between the meta consent and the broad consent models that remedies what Manson considers a main problem of the meta consent model: it's price. Manson is convinced that meta consent may be costlier than broad consent. We have previously acknowledged that this might be case due to the features above, but given that meta consent solves the expiry problem - and generally is more sensitive to individual consent preferences - we believe that biobanks have an imperfect duty to accept such costs. Assessing the added costs is notoriously difficult. After all it is an empirical question. It is worth noting, however:

5) National level: Meta consent is a model to be implemented at national level. It concerns all biobanks and all data sources in a society.

An individual biobank could decide to implement a meta consent system, but if meta consent is implemented digitally at national level as we propose, the costs at individual biobank or research project level is likely to be insignificant.

We have, contra Manson in this and previous articles not argued that research participants have a right to design future consent requests, i.e. to provide meta consent. We have, however, shown 
that meta consent is an ethically better solution than alternative models of consent. In the absence of any strong reasons not to prefer an ethically better solution, we remain convinced that meta consent is the consent model for the future. 


\section{References}

